\title{
The conditions for star formation at low metallicity: results from the LMC
}

\author{
J. M. Oliveira ${ }^{1}$, J. Th. van Loon $^{1}$ and S. Stanimirović ${ }^{2}$ \\ ${ }^{1}$ Astrophysics Group, Keele University, UK; ${ }^{2}$ Radio Astronomy Lab, UC Berkeley, USA
}

\begin{abstract}
We present our recent work on the conditions under which star formation occurs in a metal-poor environment, the Large Magellanic Cloud $([\mathrm{Fe} / \mathrm{H}] \sim-0.4)$. Water masers are used as beacons of the current star formation in Hil regions. Comparing their location with the dust morphology imaged with the Spitzer Space Telescope, and additional $\mathrm{H} \alpha$ imaging and groundbased near-infrared observations, we conclude that the LMC environment seems favourable to sequential star formation triggered by massive star feedback (Oliveira et al. 2006). Good examples of this are 30 Doradus and N 113. There are also H II regions, such as N 105A, where feedback may not be responsible for the current star formation although the nature of one young stellar object (YSO) suggests that feedback may soon start making an impact. The chemistry in one YSO hints at a stronger influence from irradiation effects in a metal-poor environment where shielding by dust is suppressed (van Loon 2005).
\end{abstract}

Keywords. stars: formation; Magellanic Clouds; HII regions; circumstellar matter

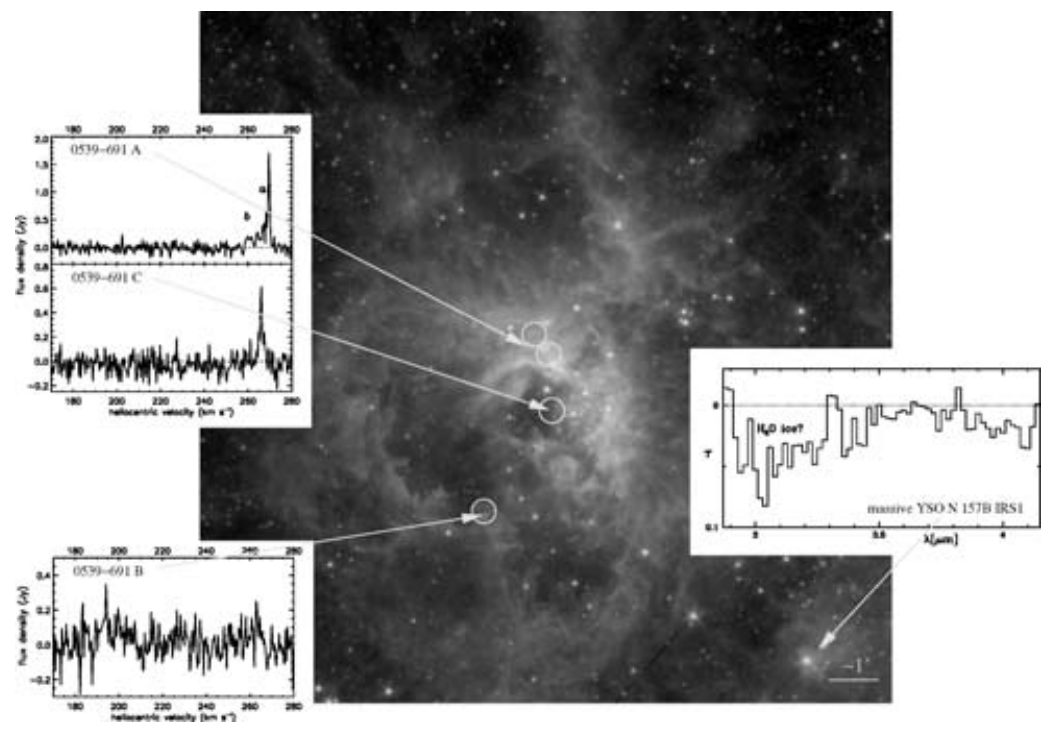

Figure 1. Spitzer $3.6+4.5+8 \mu \mathrm{m}$ composite image of 30 Doradus, showing sites of massive star formation. Water masers (l) pinpoint ongoing star formation; the ISAAC/VLT spectrum of the massive YSO (r) shows a hint of water ice. More details on this and other H II regions in the LMC can be found in van Loon et al. (2005) and Oliveira et al. (2006).

\section{References}

Oliveira, J.M. et al., 2006, MNRAS in press, astro-ph/0609036

van Loon, J.Th. et al., 2005, MNRAS 364, L71 